\title{
Implementation of polarization agility in planar phased-array antennas by means of interleaved subarrays
}

\begin{abstract}
Massimiliano Simeoni, ${ }^{1}$ Ioan E. Lager, ${ }^{1}$ Cristian I. Coman, ${ }^{2}$ and Antoine G. Roederer ${ }^{1}$

Received 9 March 2009; revised 14 July 2009; accepted 21 July 2009; published 21 October 2009.

[1] A method for ensuring polarization agility in conjunction with beam steering in planar array antennas is proposed. It relies on interleaving two subarrays with orthogonal, linear polarizations that, together, can generate arbitrary polarization states: adjustable linear, elliptical, and left- or right-handed circular. The complexity of the resulting system is comparable with that of standard, fully populated array antennas consisting of identical, linearly polarized elements. By dynamically controlling the amplitude and the phase of the signals fed to the subarrays, a stable polarization state can be maintained during beam steering. The concept is validated by numerically investigating an architecture obtained by interleaving nonuniform subarrays designed by means of a deterministic placement strategy. The effects of the mutual coupling between the different radiating elements are modeled and discussed.
\end{abstract}

Citation: Simeoni, M., I. E. Lager, C. I. Coman, and A. G. Roederer (2009), Implementation of polarization agility in planar phased-array antennas by means of interleaved subarrays, Radio Sci., 44, RS5013, doi:10.1029/2009RS004175.

\section{Introduction}

[2] Accounting for the polarization properties of targets is widely recognized as an instrumental ingredient for enhancing the detection probability and clutter suppression in radar systems [Giuli, 1986]. Moreover, the possibility of dynamically reconfiguring the polarization of the transmitted signal has been indicated as an instrument for reducing the glint error in tracking radar systems [Skolnik, 1981, p. 172]. In mobile radio communications, polarization diversity can be efficiently employed for reducing the effects of multipath fading [Lee, 1998, p. 391] while, recently, the ability of controlling in a flexible manner the polarization properties of the antenna front end has been identified as one of the key features required for the development of modern in-flight entertainment systems aiming at broadband multimedia applications. On these grounds, the topic of polarization agility is at the core of intense investigations within the antenna design community [see Gao et al., 2006, and references therein].

[3] It is interesting to note that the polarization agility was closely associated to array antennas already in the

\footnotetext{
${ }^{1}$ International Research Centre for Telecommunications and Radar, Delft University of Technology, Delft, Netherlands.

${ }^{2}$ NATO Consultation, Command and Control Agency, The Hague, Netherlands
}

Copyright 2009 by the American Geophysical Union. 0048-6604/09/2009RS004175\$11.00 early sixties [Nolen, 1965]. The implementation of this feature was thought of by integrating appropriate individual radiators in standard array configurations. For dynamic adjustment of the polarization state of the radiated field, actively controlled antennas seemed the natural answer. Some typical such structures are reported by Haskins et al. [1991], Haskins and Dahele [1997], and Korošec et al. [2006]. Nevertheless, this approach lead to a substantial increase in the system's complexity. In an attempt to sidestep this impediment, Zhong et al. [2004] reported an architecture consisting of 16 elements that used one single phase shifter, at the expense of having a fixed beam. A polarization-agile antenna that allowed a limited beam steering in one direction was presented by Yen and Chu [2005], but the relevant design had a reduced number of elements and a very high complexity. The complexity of the arrays above referred to is a direct consequence of the highly complicated feeding network that they require. For mitigating this drawback, Huang [1986] and Hall et al. [1989] proposed the use of radiators with a fixed polarization that are grouped in compounds with a sequential rotation of their feeding. This concept is widely employed for obtaining circular polarization, as it is the case with the recent implementation described by Jazi and Azarmanesh [2006] or the configurations discussed by Ramirez et al. [2000] and Ramirez and De Flaviis [2003]. Nevertheless, the sequential rotation yields meta-elements having large electrical dimensions that, in turn, leads to the possibility of the onset 


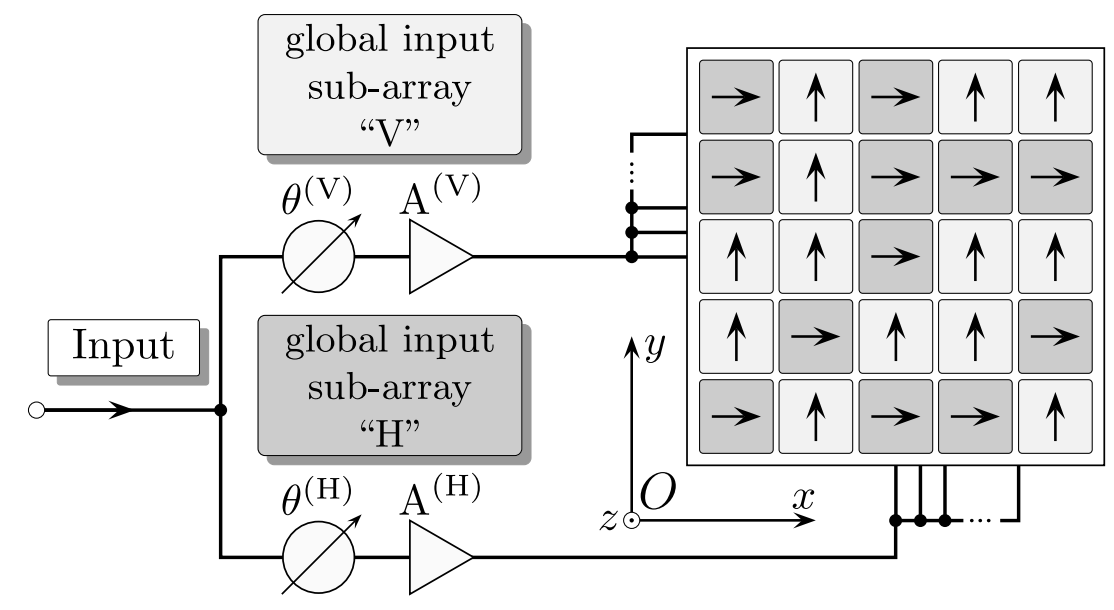

Figure 1. Array antenna system composed of two interleaved sparse arrays and the pertaining phase and amplitude control circuitry required for achieving polarization agility. The phase shifters (one per radiator) required for steering the antenna beam are not shown.

of grating lobes, especially in the diagonal plane [Hansen, 1998, p. 193].

[4] The topic of polarization-agile array antennas was approached from a completely different perspective by Simeoni et al. [2007], where the use of a shared aperture antenna consisting of interleaved subarrays with orthogonal, linear polarizations was proposed. Arbitrary linear polarization states could be generated by resorting to subarray amplitude control. In view of the elementary radiators supporting one single, linear polarization, the complexity of the required feeding network was significantly reduced while preserving very narrow radiation beams. At the same time, the conditions were created for ensuring a better isolation between the subarrays that translates into a high polarization purity of the aggregate radiated field. The solution proposed by Simeoni et al. [2007] enables the achievement of narrow radiation beam widths while keeping the complexity of the feeding network reduced. This is accomplished by accepting a trade-off in terms of antenna gain that is lower than that corresponding to uniform arrays made of polarization-agile elements.

[5] The present contribution extends the concept introduced by Simeoni et al. [2007] by demonstrating the possibility of generating arbitrary polarization states (adjustable linear, elliptical, and left- or right-handed circular) in conjunction with beam steering. The study of the effect of the mutual coupling occurring between the individual radiators in the array antenna and a discussion on the antenna physical implementation are also included.

\section{Design Philosophy}

[6] The case of a planar array antenna, consisting of radiators located on a fixed lattice (as the one depicted in
Figure 1) is considered. The analysis is henceforth confined to flanged, aperture type radiators, the flange being taken to be infinitely extended. In this configuration, position is specified by the coordinates in a Cartesian reference frame $O x y z$, the plane $x O y$ coinciding with the flange. The unit vectors $\left\{\boldsymbol{i}_{x}, \boldsymbol{i}_{y}, \boldsymbol{i}_{z}\right\}$, in this order, constitute a right-handed system. The array antenna radiates into the half-space $\{z \geqslant 0\}$, where the medium is taken to be vacuum. A polar reference frame $\operatorname{Or} \varphi \vartheta$ is employed for accounting for the electromagnetic field quantities in the far-field region. (The angle $\vartheta$ measures the tilting with respect to the $O z$ axis and $\varphi$ measures the rotation around the $O z$-axis (with the $O x$-axis being taken as reference). The triplet $\left\{\boldsymbol{i}_{\varphi}, \boldsymbol{i}_{\vartheta}, \boldsymbol{i}_{r}\right\}$ forms a right-handed system. For preventing the nondetermination occurring at $\vartheta=0$, the polar reference frame is taken in that case as $\left\{\boldsymbol{i}_{\varphi}, \boldsymbol{i}_{\vartheta}, \boldsymbol{i}_{r}\right\} \equiv\left\{\boldsymbol{i}_{x}, \boldsymbol{i}_{y}, \boldsymbol{i}_{z}\right\}$.) The radiators are fed by timeharmonic signals of identical frequency $f$. Correspondingly, the wavelength in the free space reads $\lambda=c_{0} / f$, where $c_{0}$ is the wave velocity in vacuum.

[7] It is well known [see Kraus and Marhefka, 2002; Balanis, 1997] that any of the following polarization states: linear, elliptical and left- or right-handed circular can be ascribed to the electromagnetic field, depending on the character of the electric field strength $\boldsymbol{E}$. The polarization state can be expressed in terms of two parameters: the tilting angle $\tau$ and the ellipticity angle $\varepsilon$ (see Kraus and Marhefka [2002, pp. 44-49] for the relevant definitions). The following polarization states are of particular practical interest: (1) linear, characterized by $\varepsilon=0$ and a variable $\tau$, yielding the polarization angle; (2) right-handed, circularly polarized, characterized by $\varepsilon=-45^{\circ}$ and $\tau$ being irrelevant; (3) left-handed, circularly polarized, characterized by $\varepsilon=45^{\circ}$ and $\tau$ being irrelevant. 
[8] Let now $\boldsymbol{E}$ denote the representative electric field strength in the far-field region of the array antenna. It is conjectured that any of the polarization states above enumerated can be generated by superposing the effect of two subarrays that radiate mutually orthogonal waves (generically referred to as "horizontal" ("H") and "vertical" ("V"), respectively), each of which being fed by a global input signal with predetermined amplitude and phase. Furthermore, the desired polarization state can be maintained when steering the beam to an arbitrary direction defined by the doublet $\left(\vartheta_{0}, \varphi_{0}\right)$, by dynamically controlling the same phases and amplitudes. Note that the relevant global input signals are distributed to the individual radiators by two separated power distribution networks: one for the "horizontal" subarray and one for the "vertical" subarray. At this point the standard phaseshifting strategy (one phase shifter per radiating element) is employed for achieving coherent summation in the desired direction $\left(\vartheta_{0}, \varphi_{0}\right)$ [Mailloux, 1994].

[9] These statements will be hereafter demonstrated for the case of a generic array composed of the following subarrays: (1) one consisting of $N^{(\mathrm{H})}$ identical "horizontally" polarized elements (parallel to the $O x$-axis) and generating the $E_{x}$ component of the total field; (2) one consisting of $N^{(\mathrm{V})}$ identical "vertically" polarized elements (parallel to the $O y$-axis) and generating the $E_{y}$ component of the total field (see Figure 1). The quantities pertaining to the two subarrays will be identified in the following by means of the superscripts $(\mathrm{H})$ and $(\mathrm{V})$, respectively. (In the case of including the effects of the mutual coupling, the two subarrays do not radiate waves purely polarized along the "horizontal" and the "vertical" directions. Despite this, for the sake of simplicity, the same subarrays nomenclature will be employed.) A complementary arrangement of the two subarrays and a good balance in terms of the number of elementary antennas are to be preferred for ensuring some degree of similarity of the partial radiation patterns and for optimally exploiting the available array surface.

\section{Theoretical Background}

[10] The case of a phased array consisting of $N$ aperture antennas is considered. Let the locations of the aperture centers in the $\{z=0\}$ plane be defined by the position vectors $\boldsymbol{r}_{i}=\boldsymbol{i}_{x} x_{i}+\boldsymbol{i}_{y} y_{i}(i=1, \ldots, N)$. For a beam steering in the $\left(\vartheta_{0}, \varphi_{0}\right)$ direction, the array's far-field radiation pattern in an arbitrary direction $(\vartheta, \varphi)$ can be written as [Mailloux, 1994]

$$
\boldsymbol{E}(\vartheta, \varphi)=\sum_{i=1}^{N} \alpha_{i} \boldsymbol{f}_{i}(\vartheta, \varphi) F_{i}\left(\lambda, \vartheta, \varphi, \vartheta_{0}, \varphi_{0}, \boldsymbol{r}_{i}\right)
$$

where $\alpha_{i}(i=1, \ldots, N)$ are the individual complex excitation coefficients, $\boldsymbol{f}_{i}(\vartheta, \varphi)$ denotes the electric far-field radiation pattern of the $i$ th element, and the function $F_{i}$ reads

$$
F_{i}\left(\lambda, \vartheta, \varphi, \vartheta_{0}, \varphi_{0}, \boldsymbol{r}_{i}\right)=\exp \left[j k \boldsymbol{r}_{i} \cdot\left(\boldsymbol{u}-\boldsymbol{u}_{0}\right)\right]
$$

with $j=\sqrt{-1}, k=2 \pi / \lambda$ and $\boldsymbol{u}$ and $\boldsymbol{u}_{0}$ representing the unit vectors in the $(\vartheta, \varphi)$ and $\left(\vartheta_{0}, \varphi_{0}\right)$ directions, respectively.

[11] Assume that the array consists of identical, aligned elementary radiators with radiation patterns $\boldsymbol{f}_{i}(\vartheta, \varphi)=$ $f(\vartheta, \varphi)(i=1, \ldots, N)$. This situation is representative for the case of identical rectangular apertures radiating the fundamental $\mathrm{TE}_{10}$ mode, only.

[12] Assume now that the excitation coefficients pertaining to each subarray are taken to be equal $\alpha_{m}=\alpha^{(\mathrm{H})}$ for $m=1, \ldots, N^{(\mathrm{H})}$ and $\alpha_{n}=\alpha^{(\mathrm{V})}$ for $n=1, \ldots, N^{(\mathrm{V})}$. By applying superposition, the electric field radiated by the complete array reads:

$\boldsymbol{E}(\vartheta, \varphi)=\alpha^{(\mathrm{H})} \boldsymbol{f}^{(\mathrm{H})}(\vartheta, \varphi) \sum_{m=1}^{N^{(\mathrm{H})}} F_{m}+\alpha^{(\mathrm{V})} \boldsymbol{f}^{(\mathrm{V})}(\vartheta, \varphi) \sum_{n=1}^{N^{(\mathrm{V})}} F_{n}$.

This expression is obtained by separating the contributions coming from the two subarrays and by keeping in mind that the elements composing each subarray are identical.

[13] From (2) it is evident that $F_{i}\left(\lambda, \vartheta_{0}, \varphi_{0}, \vartheta_{0}, \varphi_{0}, \boldsymbol{r}_{i}\right)=$ 1. Substituting this in (3) yields

$$
\boldsymbol{E}\left(\vartheta_{0}, \varphi_{0}\right)=\alpha^{(\mathrm{H})} \boldsymbol{E}^{(\mathrm{H})}\left(\vartheta_{0}, \varphi_{0}\right)+\alpha^{(\mathrm{V})} \boldsymbol{E}^{(\mathrm{V})}\left(\vartheta_{0}, \varphi_{0}\right)
$$

where $\boldsymbol{E}^{(\mathrm{H})}\left(\vartheta_{0}, \varphi_{0}\right)$ and $\boldsymbol{E}^{(\mathrm{V})}\left(\vartheta_{0}, \varphi_{0}\right)$ denote the aggregate far-field electric field strengths radiated by each of the two subarrays, in the scanning direction.

[14] Let now the case of an observer intercepting the total radiated far field in an arbitrary direction $(\vartheta, \varphi)$. The polarization state of the received field is measured in a local reference $O^{\prime} x^{\prime} y^{\prime} z^{\prime}$ chosen such that $\left\{\boldsymbol{i}_{x^{\prime}}, \boldsymbol{i}_{y^{\prime}}, \boldsymbol{i}_{z^{\prime}}\right\} \equiv$ $\left\{\boldsymbol{i}_{\varphi}, \boldsymbol{i}_{\vartheta}, \boldsymbol{i}_{r}\right\}$. In the far-field region $\boldsymbol{E}\left(\vartheta_{0}, \varphi_{0}\right)$ can be ascribed the value

$$
\boldsymbol{E}\left(\vartheta_{0}, \varphi_{0}\right)=\xi\left(A^{(\mathrm{H})} \boldsymbol{i}_{\varphi}+A^{(\mathrm{V})} \boldsymbol{i}_{\vartheta}\right)
$$

were the constant quantity $\xi$ has the expression

$$
\xi=1 / 2\left[\left|\boldsymbol{E}^{(\mathrm{H})}\left(\vartheta_{0}, \varphi_{0}\right)\right|+\left|\boldsymbol{E}^{(\mathrm{V})}\left(\vartheta_{0}, \varphi_{0}\right)\right|\right]
$$

and the complex parameters $A^{(\mathrm{H})}$ and $A^{(\mathrm{V})}$ will be specified below. Note that $\xi$ acts as a homogenization factor.

\section{Achieving Polarization States of Practical Interest}

[15] The expression in (5) opens the path toward obtaining arbitrary polarization states in the local refer- 
ence frame $O^{\prime} x^{\prime} y^{\prime} z^{\prime}$ by means of conveniently adjusting $\boldsymbol{E}\left(\vartheta_{0}, \varphi_{0}\right)$ :

Linear polarization at an arbitrary tilt angle $\tau$, by selecting

$$
\begin{aligned}
& A^{(\mathrm{H})}=\cos (\tau) \\
& A^{(\mathrm{V})}=\sin (\tau)
\end{aligned}
$$

Circular polarization, by selecting

$$
\begin{gathered}
A^{(\mathrm{H})}= \pm \mathrm{j} \\
A^{(\mathrm{V})}=1
\end{gathered}
$$

the minus sign corresponding to the right-hand and the plus to the left-hand polarizations, respectively.

[16] The control of $\boldsymbol{E}\left(\vartheta_{0}, \varphi_{0}\right)$ is exerted through the constants $\alpha^{(\mathrm{H})}$ and $\alpha^{(\mathrm{V})}$. By substituting (4) in (5) it is found that

$$
\begin{gathered}
\alpha^{(\mathrm{H})} E_{\varphi}^{(\mathrm{H})}\left(\vartheta_{0}, \varphi_{0}\right)+\alpha^{(\mathrm{V})} E_{\varphi}^{(\mathrm{V})}\left(\vartheta_{0}, \varphi_{0}\right)=\xi A^{(\mathrm{H})} \\
\alpha^{(\mathrm{H})} E_{\vartheta}^{(\mathrm{H})}\left(\vartheta_{0}, \varphi_{0}\right)+\alpha^{(\mathrm{V})} E_{\vartheta}^{(\mathrm{V})}\left(\vartheta_{0}, \varphi_{0}\right)=\xi A^{(\mathrm{V})} .
\end{gathered}
$$

The solution of this system of linear equations with complex coefficients reads

$$
\begin{aligned}
& \alpha^{(\mathrm{H})}=\mathrm{B}^{(\mathrm{H})} / \mathrm{D} \\
& \alpha^{(\mathrm{V})}=\mathrm{B}^{(\mathrm{V})} / \mathrm{D}
\end{aligned}
$$

in which the parameters $B^{(H)}, B^{(V)}$ and $D$ are evaluated as

$$
\begin{gathered}
\mathrm{B}^{(\mathrm{H})}=\xi\left[A^{(\mathrm{H})} E_{\vartheta}^{(\mathrm{V})}\left(\vartheta_{0}, \varphi_{0}\right)-A^{(\mathrm{V})} E_{\varphi}^{(\mathrm{V})}\left(\vartheta_{0}, \varphi_{0}\right)\right] \\
\mathrm{B}^{(\mathrm{V})}=\xi\left[A^{(\mathrm{V})} E_{\varphi}^{(\mathrm{H})}\left(\vartheta_{0}, \varphi_{0}\right)-A^{(\mathrm{H})} E_{\vartheta}^{(\mathrm{H})}\left(\vartheta_{0}, \varphi_{0}\right)\right] \\
\mathrm{D}=E_{\varphi}^{(\mathrm{H})}\left(\vartheta_{0}, \varphi_{0}\right) E_{\vartheta}^{(\mathrm{V})}\left(\vartheta_{0}, \varphi_{0}\right)-E_{\vartheta}^{(\mathrm{H})}\left(\vartheta_{0}, \varphi_{0}\right) E_{\varphi}^{(\mathrm{V})}\left(\vartheta_{0}, \varphi_{0}\right) .
\end{gathered}
$$

Note that the case when the denominator $\mathrm{D}$ is zero implies that $E_{\varphi}^{(\mathrm{H})}\left(\vartheta_{0}, \varphi_{0}\right) / E_{\vartheta}^{(\mathrm{H})}\left(\vartheta_{0}, \varphi_{0}\right)=E_{\varphi}^{(\mathrm{V})}\left(\vartheta_{0}, \varphi_{0}\right) / E_{\vartheta}^{(\mathrm{V})}\left(\vartheta_{0}, \varphi_{0}\right)$, requiring the vectors $\boldsymbol{E}^{(\mathrm{H})}\left(\vartheta_{0}, \varphi_{0}\right)$ and $\boldsymbol{E}^{(\mathrm{V})}\left(\vartheta_{0}, \varphi_{0}\right)$ to be parallel. This situation is ruled out by the initial choice of the excitations in the two subarrays.

[17] It can now be concluded that knowing the radiation patterns of the " $\mathrm{H}$ " and " $\mathrm{V}$ " subarrays is sufficient for being able to reconstruct any desired polarization state in the $\left(\vartheta_{0}, \varphi_{0}\right)$ direction. The adjustment of the polarization state is achieved by controlling two subarray complex parameters $\alpha^{(\mathrm{H})}$ and $\alpha^{(\mathrm{V})}$ (amplitude and phase) thus making possible the implementation of polarization agility.

\section{Numerical Validation}

[18] The concepts discussed in section 4 are now validated by numerically investigating the cases of a shared aperture antenna consisting of interleaved, nonuniform subarrays. The numerical experiments are carried out by means of a Matlab implementation of the formulation presented in section 4. A complementary subarray configuration is employed. The subarrays under consideration share a regular, $15 \mathrm{~mm}$ spaced, orthogonal lattice. Upon selecting an operational frequency $f=13 \mathrm{GHz}$, the chosen spacing corresponds to about $0.65 \lambda$.

[19] The elementary radiators are taken to be flanged apertures. All apertures in a subarray are identical and aligned, the radiation of the fundamental $\mathrm{TE}_{10}$ mode being accounted for, exclusively. The dimensions of all apertures are taken to be $a \times b=12 \mathrm{~mm} \times 6 \mathrm{~mm}$, the apertures in the " $H$ " subarray radiating a $\mathrm{TE}_{10}$ mode oriented along $\boldsymbol{i}_{x}$ (thus having the long-edge parallel to the $O y$-axis) and the apertures in the " $\mathrm{V}$ " subarray radiating a $\mathrm{TE}_{10}$ mode oriented along $\boldsymbol{i}_{y}$ (thus having the long-edge parallel to the $O x$-axis). The mutual coupling is, in the first instance, not accounted for. The far-field radiation patterns of the selected elementary antennas are computed in the standard manner, by means of the Fourier transform of the waveguide aperture field [see Balanis, 1997, p. 599].

[20] The radiation properties and the polarization parameters of the fields radiated by the considered shared aperture antenna are evaluated for a beam steering at $\left(\vartheta_{0}, \varphi_{0}\right)=\left(30^{\circ}, 45^{\circ}\right)$ for the cases of a tilted linear (TL) and of a right-handed circular (RHC) polarizations. The discussed far-field radiation patterns refer to the total radiated power computed as

$$
P_{\text {tot }}(\vartheta, \varphi)=\left|E_{\vartheta}(\vartheta, \varphi)\right|^{2}+\left|E_{\varphi}(\vartheta, \varphi)\right|^{2}
$$

in which $E_{\vartheta}$ and $E_{\varphi}$ denote the components of the total electric field strength given in (3).

[21] The case of the antenna obtained by interleaving subarrays generated by using a Cyclic Difference Sets (CDS) placement strategy [Leeper, 1999] is examined, the array synthesis method being elaborated upon in Appendix A. (Other placement techniques yielding a balanced, complementary division of the initial array could be adopted, providing that the related subarrays' radiation patterns exhibit an adequate degree of similarity.) This placement method allows a complementary division of the initial array into two subarrays with similar radiation properties. The complementarity of the two constituents rules out any element overlapping, while ensuring a 


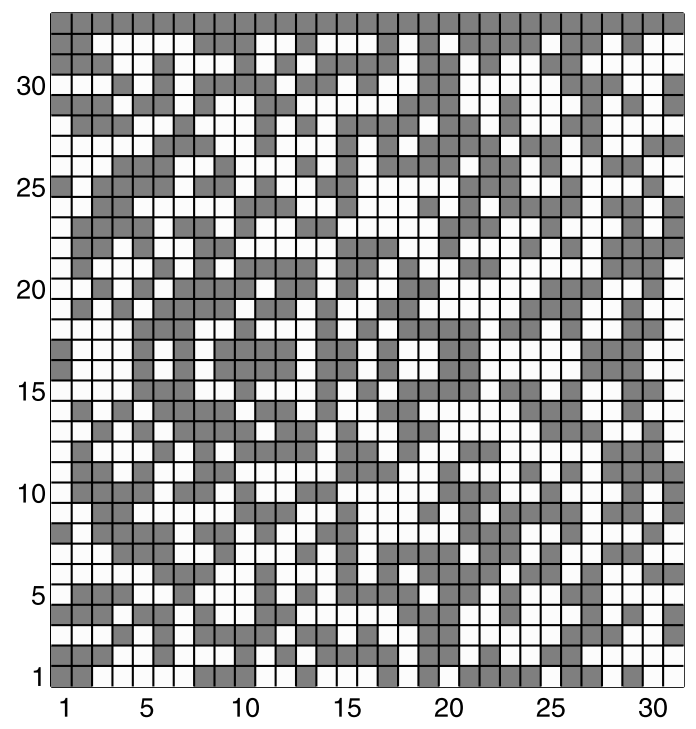

Figure 2. Architecture of the array antenna. The dark squares indicate the elements of the " $\mathrm{H}$ " subarray (consisting of $N^{(\mathrm{H})}=511$ elements), and the light ones indicate the elements of the "V" subarray (consisting of $N^{(\mathrm{V})}=512$ elements). The total number of radiating elements is $N=1023$. high degree of similarity of the partial radiation properties. The resulting array architecture is depicted in Figure 2. This choice results in an excellent balance between the number of elements in the " $\mathrm{H}$ " and " $\mathrm{V}$ " subarrays, with $N^{(\mathrm{H})}=511$ and $N^{(\mathrm{V})}=512$.

[22] The total power radiation patterns in the planes $\left\{\varphi=45^{\circ}\right\}$ and $\left\{\vartheta=30^{\circ}\right\}$ are depicted in Figures 3 and 4 , respectively. From them, it is obvious that no grating lobes are present. Moreover, additional tests, not included in this work for reason of brevity, confirm that the entire visible domain is free of grating lobes.

[23] After verifying the suitability of the radiation patterns, the numerical experiments proceed with the generation of a TL polarization with a prescribed tilting angle $\tau=20^{\circ}$ and of a RHC polarization. The polarization ellipses [see Kraus and Marhefka, 2002] representing the polarization states of the field radiated in the $\left(\vartheta_{0}, \varphi_{0}\right)$ direction are depicted in Figure 5. The effectiveness of the proposed excitation strategy is evident since the desired polarization states are exactly reproduced.

[24] To further demonstrate the configuration's capability to replicate the desired polarization states, the variation of the ellipticity obtained when a RHC polarization is prescribed is plotted in the planes $\left\{\varphi=45^{\circ}\right\}$ and $\{\vartheta=$ $\left.30^{\circ}\right\}$ in Figures 6 and 7. It can be observed that, except for a narrow angular region by and large overlapping with the main beam (see Figures 3 and 4), the ellipticity is largely different than the desired $\varepsilon=-45^{\circ}$. There is but one isolated "peak" in Figure 7 with an ellipticity

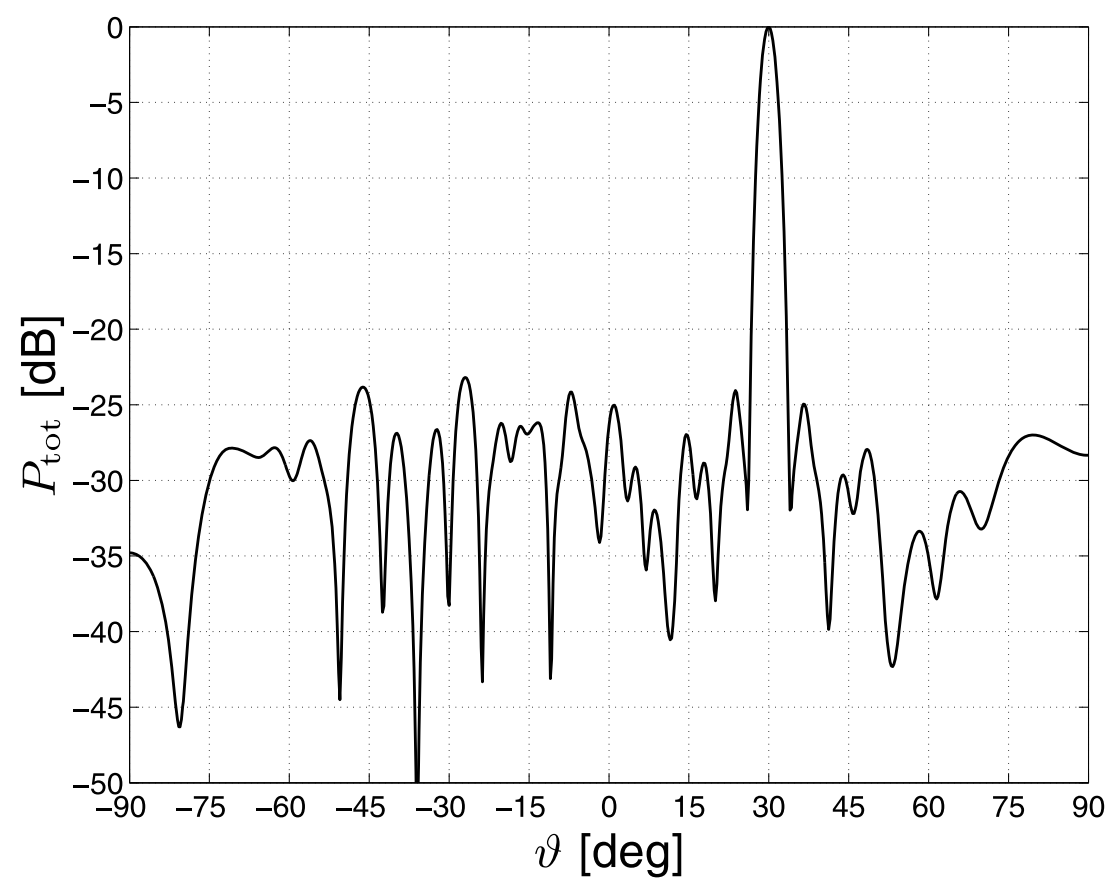

Figure 3. Array pattern in the $\left\{\varphi=45^{\circ}\right\}$ plane. 


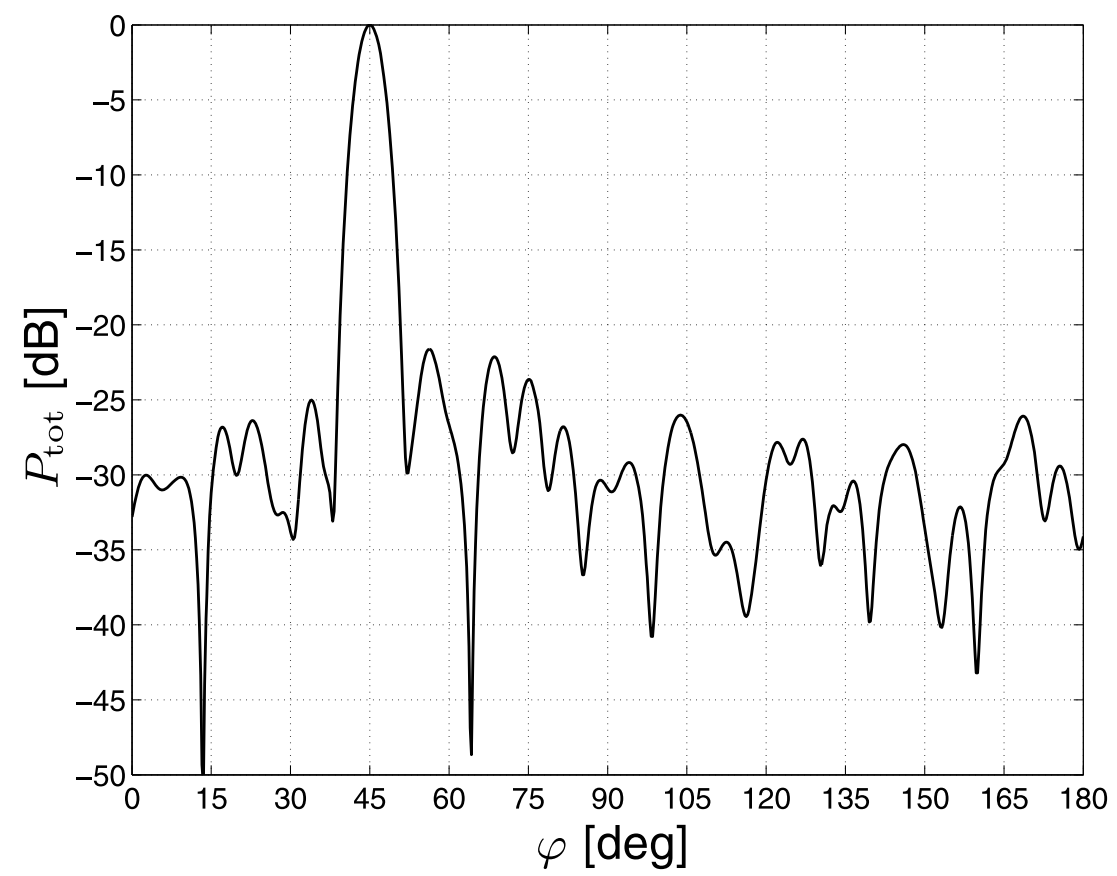

Figure 4. Array pattern in the $\left\{\vartheta=30^{\circ}\right\}$ plane.

coming close to $-40^{\circ}$. Nevertheless, this "peak" coincides with a deep null in the radiation pattern (see Figure 4) and, hence, there is virtually no power radiation in that direction. Summarizing, it can be concluded that the employed polarization synthesis procedure induces an efficacious polarization filtering. This property can be employed for substantially increasing the signal to noise ratio in the systems using antennas of the type proposed in the present work.

[25] The assessment of the robustness of the advocated polarization synthesis strategy is completed by studying the effect of frequency variations on the polarization state of the radiated field. A frequency excursion of $1 \mathrm{GHz}$ $( \pm 500 \mathrm{MHz})$ around the operational frequency of $13 \mathrm{GHz}$ causes, apart from the expected mispointing of the main beam (about $1^{\circ}$ from the desired $\left(\vartheta_{0}, \varphi_{0}\right)=\left(30^{\circ}, 45^{\circ}\right)$ direction), a marginal alteration of the prescribed polarization state. Concretely, when a RHC polarization is required, a frequency perturbation of $\Delta_{f}= \pm 500 \mathrm{MHz}$ produces an ellipticity of $\varepsilon=-43.8^{\circ}$ and a tilt angle of $\tau=\mp 3.85^{\circ}$. When a TL polarized field (with $\tau=20^{\circ}$ ) is prescribed, the same frequency perturbation yields $\varepsilon= \pm 1.06^{\circ}$ and $\tau=19.87^{\circ}$. As with any phased-array system, larger frequency variations steer the main beam further away from the desired scanning direction, this direction falling, eventually, outside the main beam. The increasingly poor radiation in the desired direction leads, inherently, to an increasingly difficult to control polarization state, an effect that can in no way be ascribed to the

chosen array synthesis approach. It can now be concluded that the proposed method is highly tolerant to frequency variations that make sense within the context of a given array architecture (i.e., lattice size and spacing), namely that maintain the desired scanning direction within the main radiation beam.

\section{Effect of the Mutual Coupling}

[26] In order to estimate the effects of the mutual electromagnetic coupling between the individual radiating elements on the antenna performance, a second smaller, array architecture is now considered. Again, a CDS-based

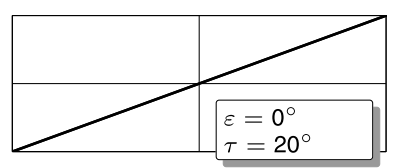

a

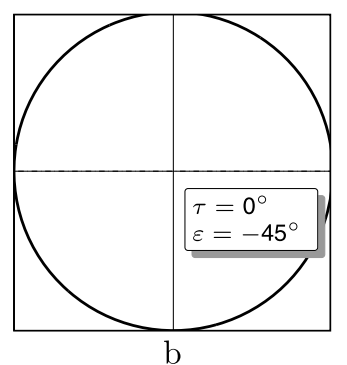

Figure 5. Polarization ellipse of the total field radiated in the direction $\left(\vartheta_{0}, \varphi_{0}\right)=\left(30^{\circ}, 45^{\circ}\right)$. (a) The case of a TL polarization with a prescribed $\tau=20^{\circ}$. (b) The case of RHC polarization. 


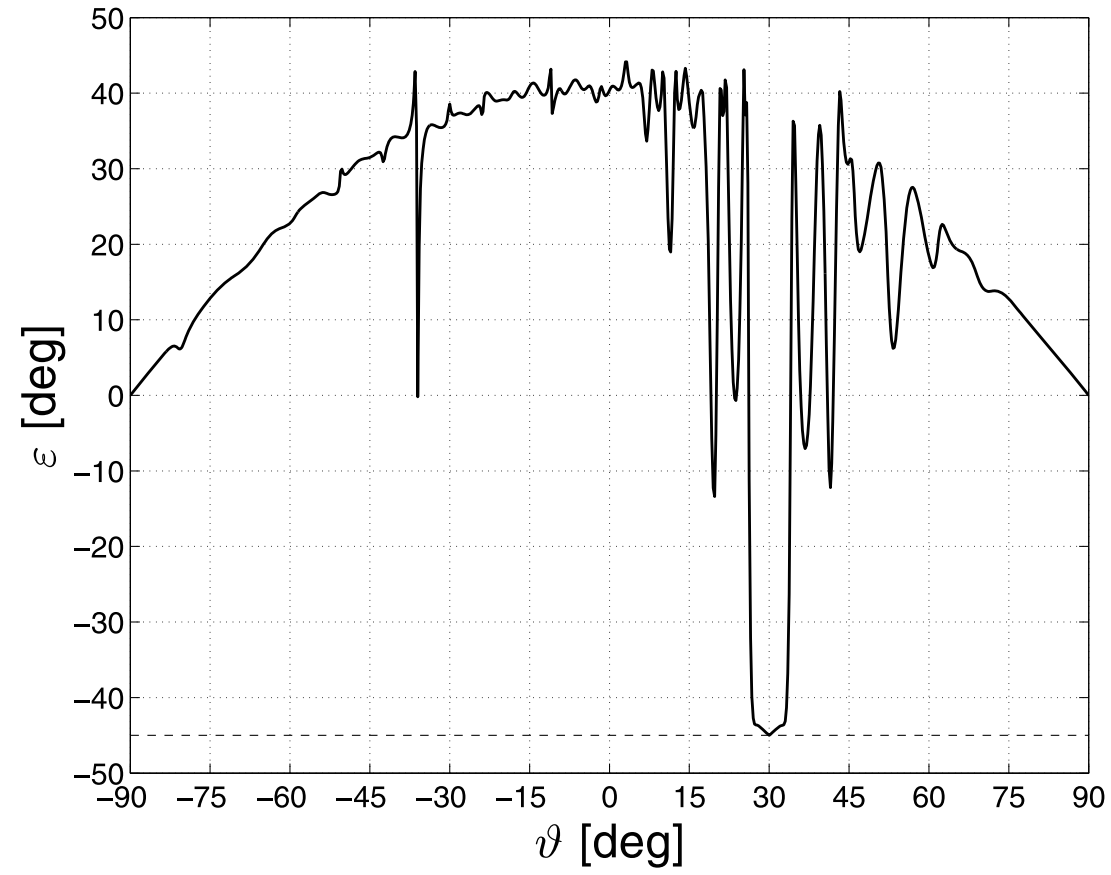

Figure 6. Variation in the $\left\{\varphi=45^{\circ}\right\}$ plane of the total field's ellipticity $\varepsilon$. The dashed line corresponds to the required $\varepsilon=-45^{\circ}$ ellipticity.

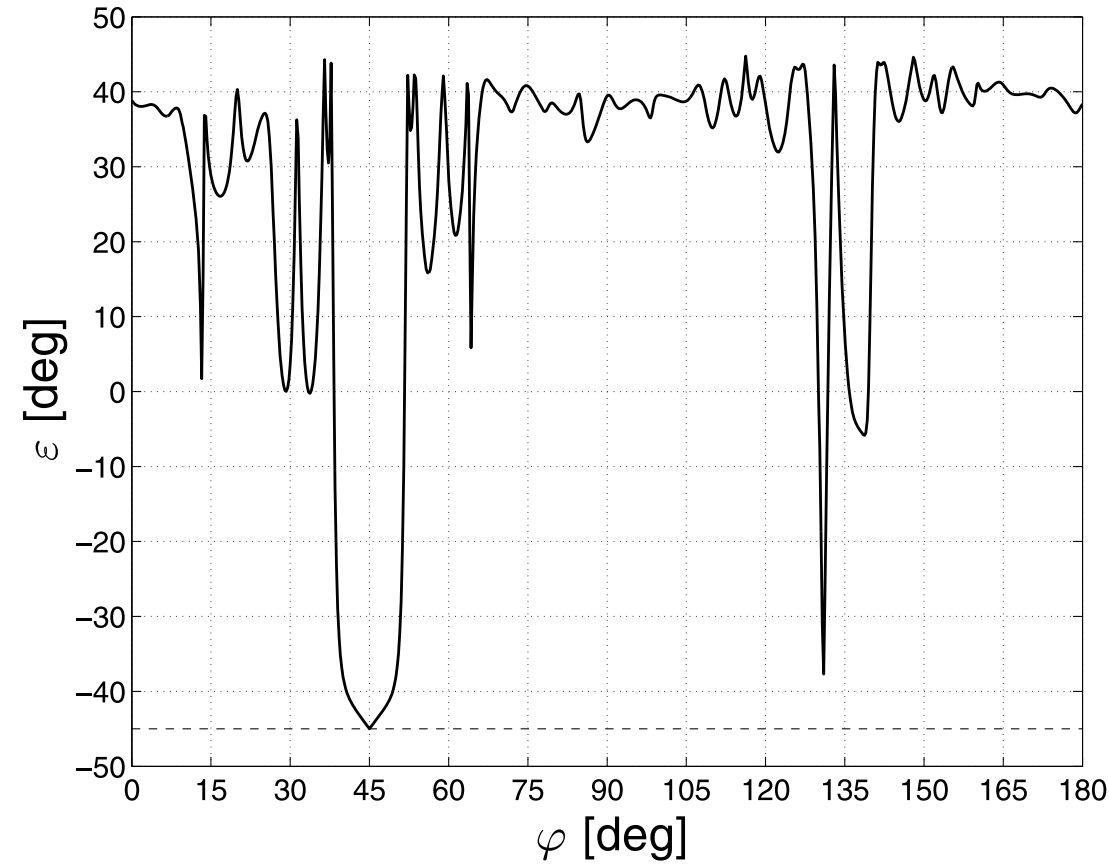

Figure 7. Variation in the $\left\{\vartheta=30^{\circ}\right\}$ plane of the total field's ellipticity $\varepsilon$. The dashed line corresponds to the required $\varepsilon=-45^{\circ}$ ellipticity. 


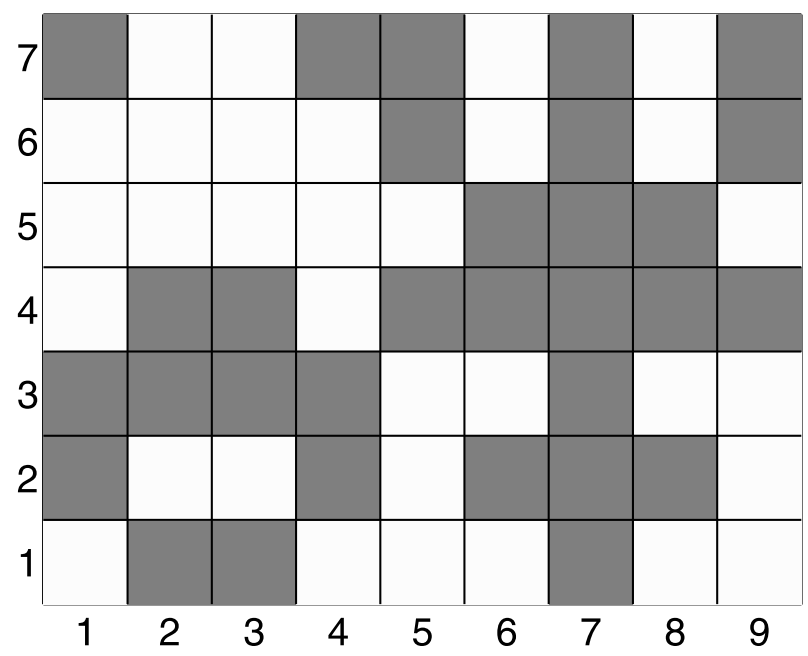

Figure 8. Architecture of the CDS $9 \times 7$ based array. The dark squares indicate the elements of the " $\mathrm{H}$ " subarray (consisting of $N^{(\mathrm{H})}=31$ elements), and the light ones indicate the elements of the " $\mathrm{V}$ " subarray (consisting of $N^{(\mathrm{V})}=32$ elements). The total number of radiating elements is $N=63$.

architecture is adopted. The $\{63,31,15\}$ CDS here employed results in a good balance between the number of elements in the two subarrays $\left(N^{(\mathrm{H})}=31\right.$ and $\left.N^{(\mathrm{V})}=32\right)$. The complete array topology, referred to as CDS $9 \times 7$, is depicted in Figure 8 .

[27] The radiating elements composing the two subarrays are taken to be open ended flanged rectangular waveguides. Two different situations are considered: (1) rectangular apertures, the cross-section dimensions of the radiating ends being: $a \times b=12 \mathrm{~mm} \times 6 \mathrm{~mm}$; (2) square apertures, the cross-section dimensions of the radiating ends being: $a \times b=12 \mathrm{~mm} \times 12 \mathrm{~mm}$.

[28] These two scenarios lead to different levels of mutual coupling between the modes of the individual apertures. In the case of the square apertures the mutual coupling is stronger and, as it will become evident in the remainder of this section, this will perturb more the polarization purity of the total radiated field.

[29] The mutual coupling between the first 15 modes (ordered with increasing cutoff wave numbers) of each radiating aperture are computed by means of the Mode Matching Method (MMM). The MMM yields a Generalized Admittance Matrix (GAM) representation of the electromagnetic coupling, as detailed by Bird [1990] and Bird and Bateman [1994]. The computational engine described by Coman et al. [2006] is employed in this work for the evaluation of the total radiated fields.

[30] Once again, the radiation properties of the CDS $9 \times$ 7 architecture are investigated for the case of the beam being scanned at $\left(\vartheta_{0}, \varphi_{0}\right)=\left(30^{\circ}, 45^{\circ}\right)$. The total power

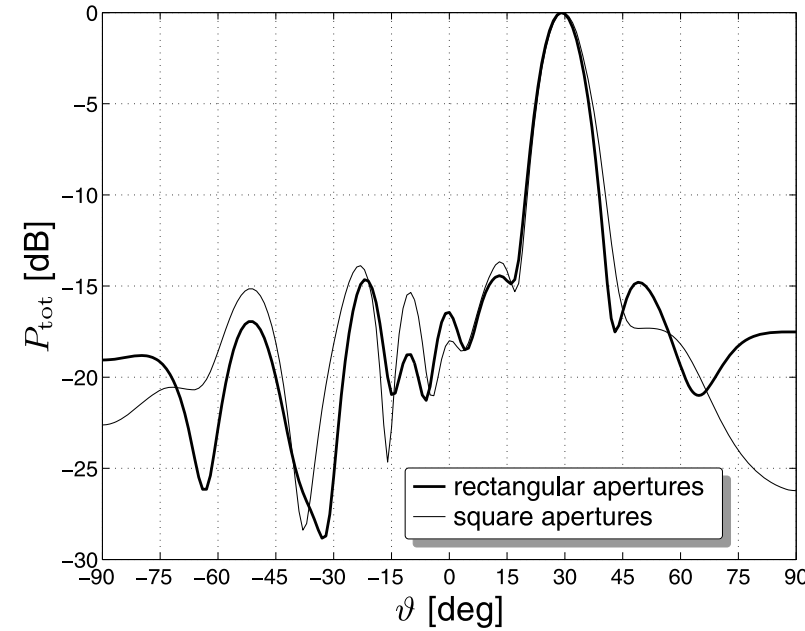

Figure 9. Array pattern of the CDS $9 \times 7$ based architecture in the $\left\{\varphi=45^{\circ}\right\}$ plane.

radiation patterns in the planes $\left\{\varphi=45^{\circ}\right\}$ and $\left\{\vartheta=30^{\circ}\right\}$ are depicted in Figures 9 and 10, respectively. The effect of the different elements' geometry and, implicitly, of the different levels of electromagnetic coupling between them, is rather marginal.

[31] Far more interesting for the scope of this work is comparing the polarization ellipses obtained in the cases of prescribing a TL polarization (with tilting angle $\tau=$ $20^{\circ}$ ) and a RHC polarization. Figure 11 depicts the polarization ellipses in the two cases of using rectangular or square radiating apertures. When using rectangular apertures the desired polarization states are well

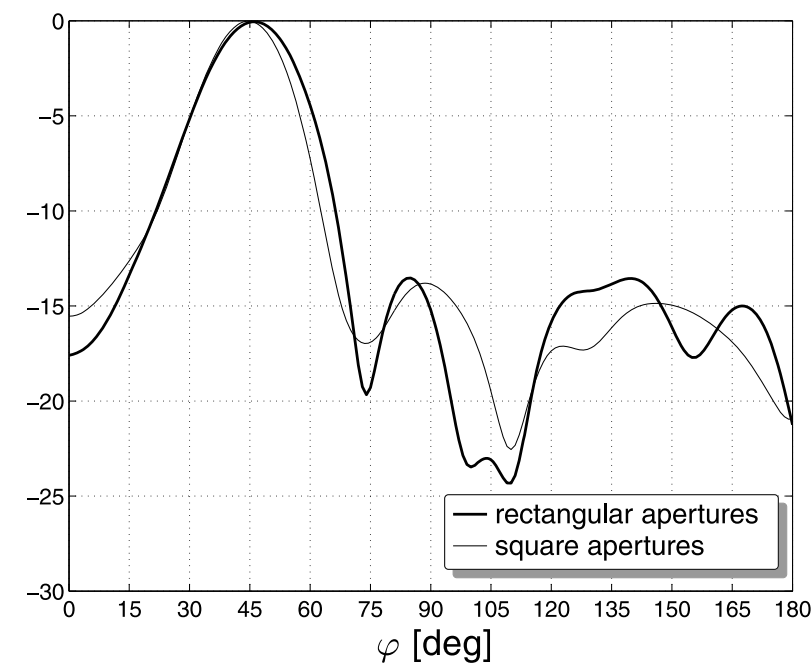

Figure 10. Array pattern of the CDS $9 \times 7$ based architecture in the $\left\{\vartheta=30^{\circ}\right\}$ plane. 


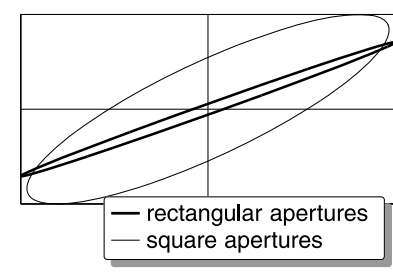

a

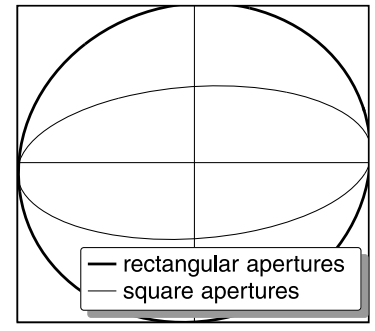

b
Figure 11. Polarization ellipse of the total field radiated in the direction $\left(\vartheta_{0}, \varphi_{0}\right)=\left(30^{\circ}, 45^{\circ}\right)$ by the CDS $9 \times 7$ based architecture using rectangular or square radiating apertures. (a) The case of a TL polarization with a prescribed $\tau=20^{\circ}$. (b) The case of RHC polarization.

approximated. As indicated above, the mutual coupling is stronger in the case of using square apertures. The stronger coupling results in a stronger excitation of the higher-order modes that can radiate waves with a different polarization than that ascribed to the relevant subarray. This, in turn, generates a degradation of the polarization state of the overall radiated field. This effect can be easily observed in Figure 11. To conclude this investigation, Figures 12 and 13 show the variation of the ellipticity in the planes $\left\{\varphi=45^{\circ}\right\}$ and $\left\{\vartheta=30^{\circ}\right\}$ obtained when a
RHC polarization is prescribed. Again, the use of rectangular apertures results in a better approximation of the desired polarization properties of the radiated wave. The polarization filtering effect observed in section 5 is also favored by the adoption of rectangular apertures.

[32] The proposed approach for generating polarization agility in scanning array antennas is robust and can, then, be used as long as the mutual coupling between the elementary antennas is kept under control.

\section{Implementation-Related Aspects}

[33] One of the major drawbacks of the presently available polarization-agile implementations is their very high (sometimes, impractical) system complexity. The overview in section 1 stressed that the majority of those array antennas employ doubly polarized elements that, moreover, in some cases also use active devices for their dynamic reconfiguration. In the case when active devices are included in the individual radiators, the already complicated power distribution network must be augmented with the relevant bias control lines.

[34] For applications in which it is acceptable to trade in some antenna gain for narrow beam widths, the proposed design strategy drastically reduces the complexity of the needed distribution network. Since the proposed implementation relies on the use of singly polarized

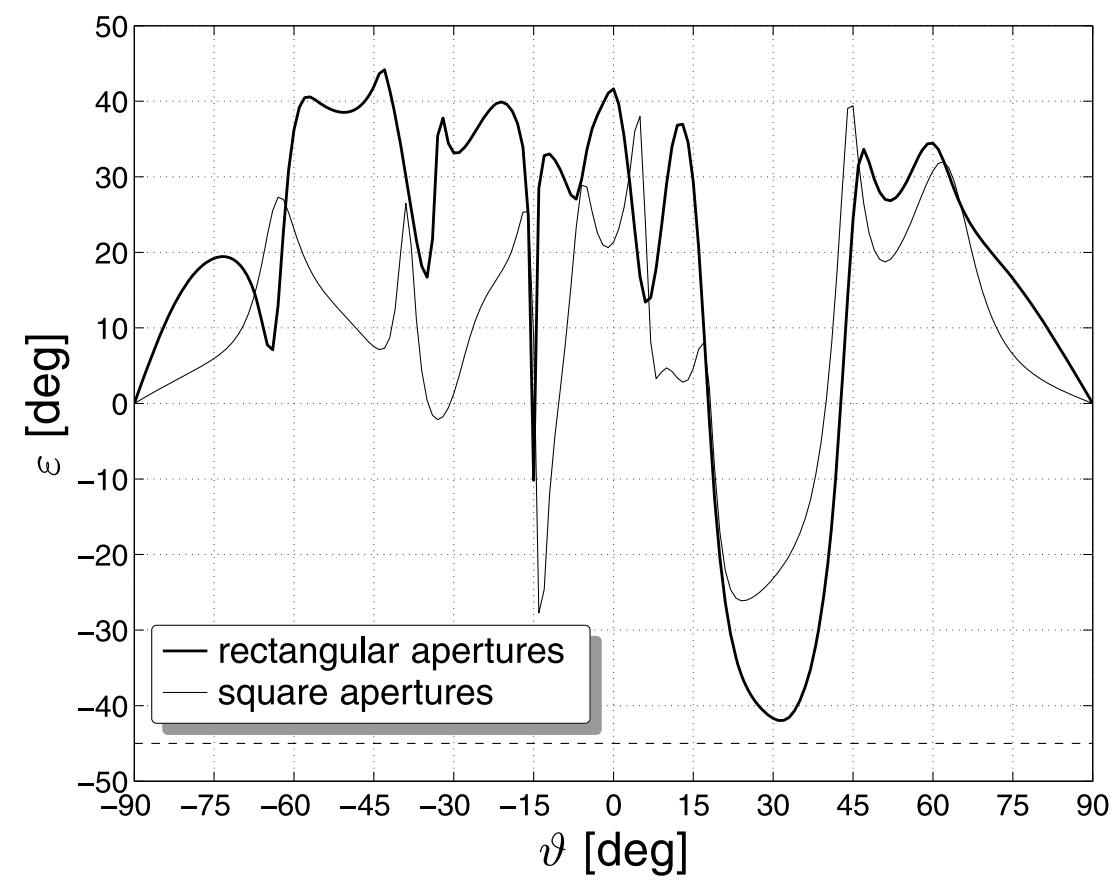

Figure 12. Variation in the $\left\{\varphi=45^{\circ}\right\}$ plane of the total field's ellipticity $\varepsilon$ in the case of the CDS $9 \times 7$ based architecture, using rectangular or square radiating apertures. The dashed line corresponds to the required $\varepsilon=-45^{\circ}$ ellipticity. 


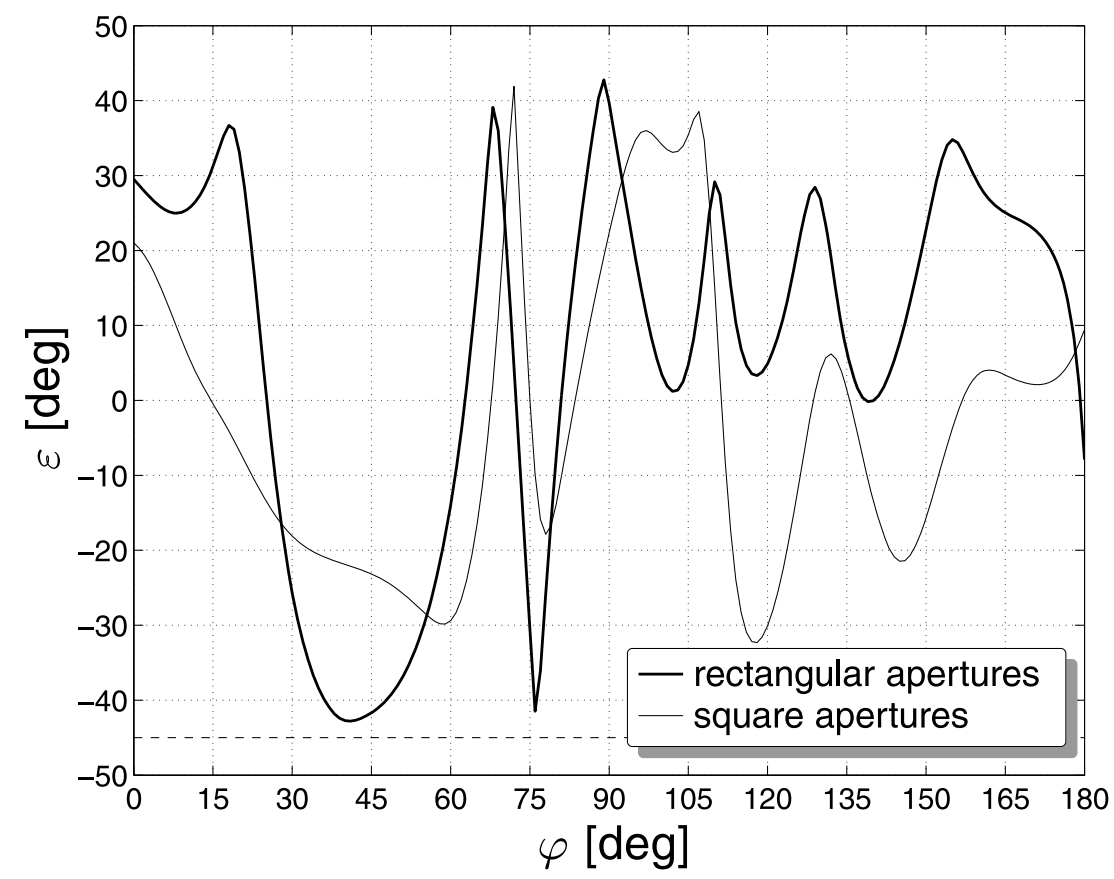

Figure 13. Variation in the $\left\{\vartheta=30^{\circ}\right\}$ plane of the total field's ellipticity $\varepsilon$ in the case of the CDS $9 \times 7$ based architecture, using rectangular or square radiating apertures. The dashed line corresponds to the required $\varepsilon=-45^{\circ}$ ellipticity.

antenna elements, every element is fed by only one signal line, instead of two. In fact, except for the additional channel phase shifters and amplifiers (see Figure 1), the complexity of the required power distribution network is similar with that of standard, fully populated, phased arrays containing the same number of elements. It is worth noting that the architecture depicted in Figure 1 lends itself to a further simplification. By examining equations $(13)-(17)$, it is clear that the coefficients $\alpha^{(\mathrm{H})}$ and $\alpha^{(\mathrm{V})}$ can be made to mimic two amplitudes and one phase difference. It then follows that one of the phase shifters in Figure 1 may be eliminated.

[35] Regarding the radiating elements, the fact that each subarray generates one single polarization implies that it can be implemented by using very simple radiators, such as square or rectangular patches, dipoles or openended waveguides. Apart from an obvious simplification of the manufacturing process for these elements, their simplicity has an additional twofold beneficial effect: on the one hand, no control of active devices is needed anymore; on the other hand, the polarization purity of the relevant elements is high that, in turn, positively influences the purity of the aggregate polarization state radiated by the array (see section 6).

[36] The fact that the individual radiation patterns are a priori known can also be exploited for avoiding the online computation of the $\alpha^{(\mathrm{H})}$ and $\alpha^{(\mathrm{V})}$ coefficients and, thus, for enhancing the beam-steering agility. To this end, equations (13) and (14) can be evaluated off-line for a (fine) division of the intended range of scanning angles, the relevant results being then stored. This allows a table look-up strategy to be employed for directly retrieving the coefficients for any desired beam-steering direction, while the array antenna is operated.

[37] When comparing the proposed strategy to the commonly used approach of assembling arrays of polarization-agile radiators it becomes evident that, when the same beam width is to be achieved, a much smaller number of phase shifters is needed to implement the polarization agility $(2$, or even 1 , instead of $2 \times N)$. It then becomes possible to adopt high-quality phase shifters with a finer phase quantization (higher number of control bits) and in turn, presumably, better system performance. In this manner, the conditions are created for a very accurate implementation of a polarization-agile system. The reduction in complexity will also positively influence the cost of such an antenna front end. It is worth noting that a further system simplification could be achieved by thinning down the number or radiating elements while preserving the occupation of the array surface. This solution would reduce the system complexity and preserve the radiation beam width at the expense of further reducing the antenna gain. In some applications, where no strict requirements are imposed on the antenna gain, thinning 


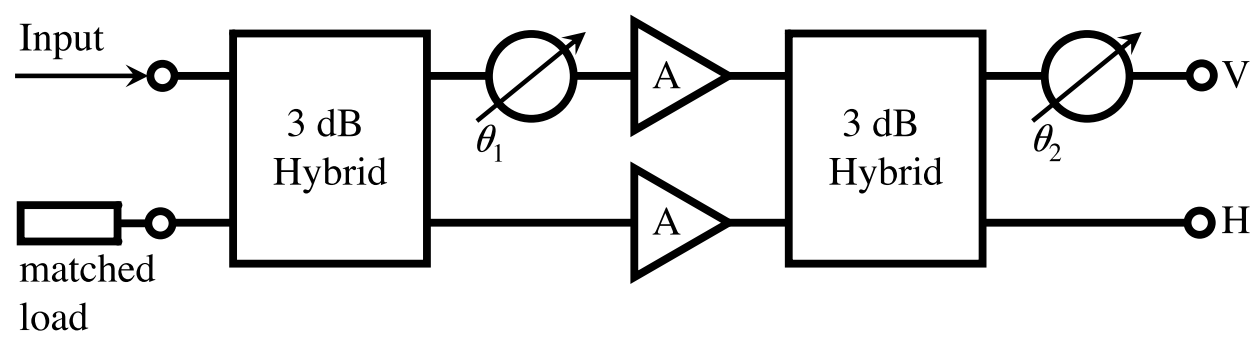

Figure 14. A possible network providing the feeding signals to the two subarrays.

the array could then be a viable solution for additional antenna system complexity reduction.

[38] Throughout the present account, deterministic placement procedures were put to use. This preference resulted in a simplification of both the optimization of the subarray radiation properties and of their interleaving. Nevertheless, it might be possible to further improve the performance of the system by resorting to stochastic placement strategies. This topic is open for investigation.

[39] To conclude with, a possible implementation of the circuitry needed for the achievement of the polarization reconfigurability is illustrated in Figure 14. The proposed network consists of two $3 \mathrm{~dB}, 90$ degree hybrids, two adjustable phase shifters and two amplifiers. One of the two inputs of the first $3 \mathrm{~dB}$ hybrid is terminated on a matched load. The two amplifiers provide a fixed amplification, this having a favorable impact on the power efficiency of these devices. Moreover, the amplifiers are located, in the signal chain, after the first phase shifter, this enables compensating for the losses introduced by this device. The output terminals (on the right-hand side of the picture) denoted as " $\mathrm{V}$ " and " $\mathrm{H}$ ", are to be connected to the vertical and horizontal power distribution networks, respectively. In the scheme displayed in Figure 14 the first phase shifter (providing a phase shift of $\theta_{1}$ radians) determines the amplitude ratio between the two output signals at the output terminals while the second one (providing a phase shift of $\theta_{2}$ radians) controls their phase difference. By adjusting the two phase shifters all polarization states can be achieved. The proof of this statement is straightforward and tedious and is omitted here for brevity. It is worth noting that, upon recalling that each individual radiator is equipped with its own phase shifter for beam scanning purposes, the function of the phase shifter $\theta_{2}$ in Figure 14 can be transferred to the beam steering ones. This leads to the elimination of one more phase shifter.

\section{Conclusions}

[40] A simple approach for implementing polarization agility in planar phased-array antennas was demonstrated.
Arbitrary polarization states: adjustable linear, elliptical and left- or right-handed circular could be obtained by interleaving two subarrays with orthogonal, linear polarizations. The stability of the generated polarization state during scanning is maintained by dynamically controlling the phase and the amplitude of the signals fed to the subarrays. The effects due to the mutual electromagnetic coupling between the individual radiators composing the arrays were modeled and discussed. Some implementation-related aspects were discussed. The complexity of the resulting system is low, being comparable with that of a standard, fully populated array antenna consisting of singly polarized elementary radiators.

\section{Appendix A: Planar Array Synthesis Using CDS Templates}

[41] The placement strategy follows closely the methodology described by Leeper [1999] that, in turn, was based on the algorithm originally described by MacWilliams and Sloane [1975]. Note that the application of this algorithm yields arrays characterized by a two-valued autocorrelation [Leeper, 1999].

[42] By starting from a CDS $D$ of parameters $\{V, K, \Lambda\}$ [Leeper, 1999], a $N_{1} \times N_{2}$ incidence matrix $M$ is assembled according to the rule

$$
m_{i\left(\bmod N_{1}\right), i\left(\bmod N_{2}\right)}= \begin{cases}1 & \text { if } i \in D \\ 0 & \text { otherwise }\end{cases}
$$

with $m_{i, j}\left(i=1, \ldots, N_{1}\right.$ and $\left.j=1, \ldots, N_{2}\right)$ denoting the elements of $M$, providing that $N_{1} N_{2}=V$ and that $N_{1}$ and $N_{2}$ are prime with respect to each other. As concerns the CDS template $D$, a large collection of such sets is readily available (D. Gordon, La Jolla cyclic difference set repository, http://www.ccrwest.org/di(r)sets.html). Nonetheless, the limited amount of available $D$-s, in conjunction with the severe conditions on $N_{1}$ and $N_{2}$, imposes nonnegligible restrictions on the possibility to synthesize 
planar arrays based on CDS templates. In particular, no square array can be designed in this manner.

\section{References}

Balanis, C. (1997), Antenna Theory, Analysis, and Design, 2nd ed., John Wiley, New York.

Bird, T. S. (1990), Analysis of mutual coupling in finite arrays of different-sized rectangular waveguides, IEEE Trans. Antennas Propag., 38(2), 166-172.

Bird, T. S., and D. G. Bateman (1994), Mutual coupling between rotated horns in a ground plane, IEEE Trans. Antennas Propag., 42(7), 1000-1006.

Coman, C. I., I. E. Lager, and L. P. Ligthart (2006), The design of shared aperture antennas consisting of differently sized elements, IEEE Trans. Antennas Propag., 54(2), 376-383.

Gao, S., A. Sambell, and S. S. Zhong (2006), Polarization-agile antennas, IEEE Antennas Propag. Mag., 48(3), 28-37.

Giuli, D. (1986), Polarization diversity in radar, Proc. IEEE, 74(2), 245-269.

Hall, P. S., J. S. Dahele, and J. R. James (1989), Design principles of sequentially fed, wide bandwidth, circularly polarised microstrip antennas, IEE Proc. H. Microwaves Antennas Propag., 136(5), 381-389.

Hansen, R. C. (1998), Phased Array Antennas, John Wiley, New York.

Haskins, P. M., and J. S. Dahele (1997), Four-element varactor diode loaded polarization-agile microstrip antenna array, Electron. Lett., 33(14), 1186-1187.

Haskins, P. M., J. S. Dahele, and P. S. Hall (1991), Active patch antenna element with diode tuning, Electron. Lett., 27(20), $1846-1848$.

Huang, J. (1986), A technique for an array to generate circular polarization with linearly polarized elements, IEEE Trans. Antennas Propag., 34(9), 1113-1124.

Jazi, M. N., and M. N. Azarmanesh (2006), Design and implementation of circularly polarised microstrip antenna array using a new serial feed sequentially rotated technique, IEE Proc., Microwaves Antennas Propag., 153(2), 133-140.

Korošec, T., P. Ritoš, and M. Vidmar (2006), Varactor-tuned microstrip-patch antenna with frequency and polarisation agility, Electron. Lett., 42(18), 7-8.
Kraus, J. D., and R. J. Marhefka (2002), Antennas: For All Applications, McGraw-Hill, New York.

Lee, W. C. Y. (1998), Mobile Communications Engineering: Theory and Applications, 2nd ed., McGraw-Hill, New York.

Leeper, D. G. (1999), Isophoric arrays-Massively thinned phased arrays with well-controlled sidelobes, IEEE Trans. Antennas Propag., 47(12), 1825-1835.

MacWilliams, F. J., and N. J. Sloane (1975), Pseudo-random sequences and arrays, Proc. IEEE, 64(12), 1715-1729.

Mailloux, R. J. (1994), Phased Array Antenna Handbook, Artech House, Norwood, Mass.

Nolen, J. (1965), Phased array polarization agility, IEEE Trans. Antennas Propag., 13(5), 820-821.

Ramirez, R. R., and F. De Flaviis (2003), A mutual coupling study of linear and circular polarized microstrip antennas for diversity wireless systems, IEEE Trans. Antennas Propag., 51(2), 238-248.

Ramirez, R. R., F. De Flaviis, and N. G. Alexopoulos (2000), Single-feed circularly polarized microstrip ring antenna and arrays, IEEE Trans. Antennas Propag., 48(7), 1040-1047.

Simeoni, M., I. E. Lager, and C. I. Coman (2007), Interleaving sparse arrays: A new way to polarization-agile array antennas?, IEEE Antennas Propag. Symp., 3145-3148.

Skolnik, M. I. (1981), Introduction to Radar Systems, 2nd ed., McGraw-Hill, New York.

Yen, S.-C., and T.-H. Chu (2005), A beam-scanning and polarization-agile antenna array using mutually coupled oscillating doublers, IEEE Trans. Antennas Propag., 53(12), $4051-4057$

Zhong, S.-S., X.-X. Yang, and S.-C. Gao (2004), Polarizationagile microstrip antenna array using a single phase-shift circuit, IEEE Trans. Antennas Propag., 52(1), 84-87.

C. I. Coman, NATO Consultation, Command and Control Agency, NL-2597 AK The Hague, Netherlands.

I. E. Lager, A. G. Roederer, and M. Simeoni, International Research Centre for Telecommunications and Radar, Delft University of Technology, NL-2628 CD Delft, Netherlands. (m.simeoni@tudelft.nl) 